14.

\section{THE EFFECTS OF A COMPUTER-BASED DRIVING GAME ON HYPOGLYCEMIA EDUCATION AMONG ADOLESCENTS WITH TYPE-1 DIABETES}

Nathan Stupiansky, $\mathrm{PhD}^{3}$, Anthony Faiola, $\mathrm{Ph} \mathrm{D}^{2}$. Joseph Defazio, PhD Instructional Systems

Technology, MS Applied Computer Technology, BS Applied Computer Technology, BA Music Performance ${ }^{1}$, Donald Orr, $M D^{3}$. ${ }^{1}$ Indiana University ${ }^{2}$ Indiana University - School of Informatics ${ }^{3}$ Indiana University School of Medicine

Purpose: Individuals with type 1 Diabetes Mellitus(T1DM) are at high risk for hypoglycemia (HG); HG while driving may result in serious accidents. Failure to perform self-blood glucose monitoring (SBGM) before driving and inadequate knowledge about preventive recommendations increases the risk of $\mathrm{HG}$ while driving, and is a particular concern for adolescents. The current study piloted an innovative computerbased driving game to increase awareness of risks and inform adolescents of current safety recommendations.

Methods: Ninety-nine participants (14-16 years) with T1DM were recruited from adolescent diabetes clinics to participate in a game-based education program about HG, which included driving modules designed to simulate the effects of hypoglycemia on vehicle operator motor skills. Participants completed a short baseline (T1) pre- and post-task questionnaire about HG which queried them on the areas of driving behaviors, HG history, driving history, SBGM habits, and self-efficacy regarding hypoglycemia awareness. A short follow-up questionnaire (T2) queried hypoglycemia knowledge and intention to engage in behaviors to reduce the chance of hypoglycemic episodes while driving. Paired sample $t$ tests were used to assess changes in HG knowledge variables.

Results: Almost all adolescents reported morning HG as the most frequent time of HG 62\% (61/99) before breakfast and $31 \%$ (31/99) after breakfast, but before lunch. Only 59\%(31/99) performed SBGM four or more times daily as recommended; $22 \%(22 / 99)$ tested twice daily or less. Almost a quarter $(23 \%$, 20/86) had an episode of severe HG in the past 3 months. Over half $(53 \%, n=52)$ of adolescents reported driving every day. $26 \%(n=26)$ did not drive, $21 \%(n=21)$ drove up to three times a week or less. Of those who drive, $41 \%(30 / 73)$ either agreed or strongly agreed that they drive when their blood glucose is $<90 \mathrm{mg} / \mathrm{dL}$ recommended as safe and $59 \%$ (43/73) reported experienced mild HG at while driving at least once. Twenty-six percent (26/99) thought that HG was not serious at all. Following completion of the driving-game module, the percentage of the sample that correctly answered each of the HG knowledge variables was significantly higher than at baseline: lowest safe driving blood glucose level $(34 \% \mathrm{~T} 1,81 \% \mathrm{~T} 2 ; p<.001)$, intention to test glucose before driving $(69 \% \mathrm{~T} 1,92 \% \mathrm{~T} 2 ; p .001)$. what to do if blood glucose feels "low" before driving $(79 \% \mathrm{~T} 1$. $92 \% \mathrm{~T} 2 ; p<.01)$, and what to do if blood glucose feels "low" while driving $(79 \% \mathrm{~T} 1,90 \% \mathrm{~T} 2 ; p<.01)$.
Conclusions: A computer driving-game intervention designed to simulate the effects of $\mathrm{HG}$ on motor skills was very effective in increasing both short-term knowledge about HG and intentions to increase HG prevention behaviors. Given the limited time for clinicians to interact with adolescent patients, gamebased education modules may be an efficient way to teach adolescents with T1DM, important skills to prevent HG while driving, as well as reduce the risk of driving-related negative health outcomes.

15.

A LONGITUDINAL STUDY OF HEALTH-COMPROMISING BEHAVIORS AMONG EMERGING ADULTS WITH TYPE 1 DIABETES DURING THE TRANSITION OUT OF HIGH SCHOOL Linda Scott, BS, $M S^{3}$, Nathan Stupiansky, $P h D^{1}$. Michael Weaver, PhD, RN ${ }^{3}$. J. Dennis

Forten berry, $M D, M S^{i}$, Timothy Stump, $M A^{2}$. Kathleen Hanna, $P h D, R N^{3}$. ${ }^{1}$ Indiana University School of Medicine ${ }^{2}$ Indiana University School of Medicine, Division of Biostatistics ${ }^{3}$ Indiana University School of Nursing

Purpose: The transitions out of high school and parental home may increase vulnerability to health for emerging adults with type 1 diabetes. Alcohol use, smoking, and marijuana use are health-compromising behaviors, which typically increase as adolescents achieve greater autonomy, but may have unique negative health consequences for young people with diabetes. The purpose of the current study is to describe involvement in health-compromising behaviors among emerging adults with type 1 diabetes post high school graduation, and to assess how involvement changes with independence from living at home.

Methods:As part of a larger longitudinal study of Type 1 diabetes self-management during the transition from high school to posthigh school, this report is of 75 emerging adults with diabetes (M $=18.3$ years, $\mathrm{SD}=.40 ; 60 \%$ female) recruited as high school seniors (T1) and with completed follow-up surveys in the fall post-high school graduation (T2). Three outcome variables (alcohol use, smoking, marijuana use [assessed at T1 and T2]) were constructed based on the responses to their respective items (i.e., During the past three months, how often did you drink alcohol?). Responses were dichotomized to classify those who did not engage in the behavior at all with those who reported any occurrences. A fourth outcome variable was constructed as a summary of each of the three behaviors to represent "any risk behavior." Living situation was assessed at T2 and dichotomized as living independently of parents or not. In order to determine the relationship of living situation to health-compromising behaviors at $\mathrm{T} 2$, Fisher's exact test was used to analyze the rates of adoption of each behavior for adolescents that had reported abstaining at T1. Results: At T1, the mean duration since diabetes diagnosis was 8.5 years $(S D=4)$ and $55 \%(41 / 75)$ were using an insulin pump. At T2, 88\% (66/75) of participants were enrolled in college, and $55 \%$ (41/75) lived independent of their parents. Health-compromising behaviors occurred at a higher prevalence about 6 months post-high school than at baseline: the portions using alcohol

Stupiansky, N., Faiola, A., Defazio, J., \& Orr, D. (2011). 14. The Effects of a Computer-Based Driving Game on Hypoglycemia Education Among Adolescents with Type-1 Diabetes. Journal of Adolescent Health, 48(2), S24-S25.

http://dx.doi.org/10.1016/j.jadohealth.2010.11.058 